\title{
PONKCS FOR QPA IN PHARMACEUTICAL INGREDIENTS
}

\author{
de Salvi, S. T. B. ${ }^{*}{ }^{*}$; Perrucci, N. F. ${ }^{1}$; Antonio, S. G. ${ }^{1}$;Paiva-Santos, C. O. ${ }^{1}$ \\ ${ }^{1}$ Department of Physical-Chemistry, Institute of Chemistry (Univ. Estadual Paulista - UNESP), Araraquara, São Paulo, Brazil
}

*simonebonemer@yahoo.com.br

In the absence of a known crystal structure, the quantitative phase analysis (QPA) cannot be done through the Rietveld method (RM). In these situations the methodology described by Scarlet and Madsen and known as PONKCS (Partial Or No Known Crystal Structure) can be as efficient as the Rietveld method. Even for cases of high peak overlap, like pharmaceuticals, the QPA can be applied satisfactorily, mainly if the crystal structure is partially known, i.e., only the unit cell parameters and space group are known. We use simulations of a mixture of two polymorphs of norfloxacin, to show that the results of the Rietveld method and PONKCS provide the same results, since we take very care to not refine unit cell in the beginning of the refinement. If preferred orientation is present in the sample used for pattern decomposition through the Pawley or Le Bail method, or in the mixture, it is possible correct it in the mixture after correction of the unit cell of the unknown phase. One well known problem with the Rietveld method in the QPA of pharmaceuticals is the atom displacement when high angle data is provided. Try to correct it can be very confusing in Rietveld method. It is an advantage for PONKCS, since the thermal displacement is not a parameter. Anyway, for copper radiation data up to $35^{\circ}(2 \mathrm{q})$ the scattering factor for the atoms do not change significantly to cause a significant change in the scale factor and then PONKCS and Rietveld methods are similar. 